\title{
TEMPERATURA-BASE, GRAUS-DIA E DURAÇÃO \\ DO CICLO PARA CULTIVARES DE TRITICALE ${ }^{(1)}$
}

\author{
MÁRIO JOSÉ PEDRO JÚNIOR ${ }^{(2,5)}$; MARCELO BENTO PAES DE CAMARGO ${ }^{(2,5)}$; \\ ADRIANA VIEIRA DE CAMARGO MORAES ${ }^{(3)}$; JOÃO CARLOS FELÍCIO ${ }^{(2)}$; \\ JAIRO LOPES DE CASTRO ${ }^{(4)}$
}

\begin{abstract}
RESUMO
Foram determinadas para as cultivares de triticale: CEP 15, IAC 2 e IAC 3, a temperatura-base, a soma térmica, expressa em graus-dia, para o período semeadura-maturação e a influência da temperatura na duração do ciclo (semeadura-maturação) da cultura na região de Capão Bonito (SP) durante os anos de 1992 a 1995. Os valores de temperatura-base variaram entre 8 e $10{ }^{\circ} \mathrm{C}$ e as somas térmicas, em graus-dia, entre: 1.048 e 1.263, em função da cultivar de triticale. A duração do ciclo do triticale nos plantios efetuados em épocas mais frias aumentou em vista da diminuição da temperatura do ar.
\end{abstract}

Palavras-chave: triticale, fenologia, soma térmica, temperatura-base, graus-dia.

\section{ABSTRACT \\ BASE-TEMPERATURE, GROWING DEGREE-DAYS AND CROP GROWTH CYCLE DURATION OF TRITICALE CULTIVARS}

Base temperature, growing degree-days and cycle growth length were evaluated at Capão Bonito, São Paulo State (Brazil), during the years from 1992 to 1995, for three triticale cultivars: CEP 15, IAC 2 and IAC 3. The obtained values of base temperature varied from 8 to $10{ }^{\circ} \mathrm{C}$ and the amount of growing degree-days required for the triticale cultivars to complete their cycle varied from 1048 to e 1263, according to the cultivar. The duration of the emergency-flowering growth stage varied from 50 to 63 days, according to the cultivar, while the duration of the flowering-maturation (harvest) growth stage was 70 days for the three cultivars analised. The low air temperature enlarged the length of the total cycle for the triticale cultivars.

Key words: triticale, phenology, base-temperature, growth stage and growing degree-days.

(1) Recebido para publicação em 17 de outubro de 2002 e aceito em 6 de agosto de 2004.

$\left(^{2}\right)$ Instituto Agronômico (IAC/APTA/SAA), Caixa Postal 28, 13001-970 Campinas (SP).

$\left({ }^{3}\right)$ EMBRAPA Milho e Sorgo, Caixa Postal 151, 35701-970 Sete Lagoas (MG).

(4) Pólo de Desenvolvimento Regional do Sudoeste Paulista (DDD/APTA/SAA), Caixa Postal 62, 18300-000 Capão Bonito (SP).

$\left({ }^{5}\right)$ Bolsista do CNPq. 


\section{INTRODUÇÃO}

O triticale é uma planta rústica originalmente utilizada para produzir farinha a ser adicionada ao trigo na panificação, e hoje seu uso está mais voltado para alimentação animal por apresentar teor de proteína superior ao do milho. É cultivado no inverno, época em que as terras estão ociosas, protege o solo contra a erosão e sua palhada abafa as plantas invasoras, possibilitando posterior cultivo mínimo de outras culturas (TAGLIARI, 1996).

O triticale é um cereal de estação fria proveniente do cruzamento do trigo e centeio (Mundstock, 1983) e sua utilização pelos agricultores tem sido feita, no Estado de São Paulo, principalmente, aproveitando regiões marginais ao cultivo do trigo, por apresentar rendimentos elevados, resistência a doenças e tolerância à acidez do solo (BAYER, 1995).

O comportamento agronômico de cultivares de trigo e triticale, no Estado de São Paulo, em condições de sequeiro e irrigado, foi avaliado por FELICIO et al., (1990 e 1993), mostrando sua adaptação regional e potencial de utilização pelos produtores paulistas. Ainda, Felicio et al. (1999) estudando épocas de semeadura do triticale, em Capão Bonito (SP), verificaram a viabilidade de seu cultivo na região, com rendimento médio de $2.200 \mathrm{~kg}$. ha ${ }^{-1}$, para semeaduras efetuadas no início de abril. Observaram, também, existir influência das diferentes épocas de semeadura, de março a maio, na duração do ciclo do triticale.

A duração do ciclo das cultivares de cereais de estação fria, cultivadas no Sul do Brasil, tem-se mostrado variável, principalmente devido à ação da temperatura do ar. O fotoperíodo tem influência em genótipos sensíveis; outros fatores ambientais exercem menor efeito sobre o desempenho agronômico das espécies (MundSTOCK, 1983).

Os cultivares de trigo no País apresentam pouca ou nenhuma resposta ao fotoperíodo e os processos de desenvolvimento das plantas são controlados, principalmente, pela temperatura (Rodrigues, 2000). Da mesma maneira, as cultivares de triticale, por meio de melhoramento genético, perderam a alta sensibilidade ao fotoperíodo (Mundstock, 1983), fazendo com que a temperatura seja o fator de maior influência na duração do ciclo. Segundo BRUNinI et al. (1976), a temperatura do ar é o principal elemento a influenciar o desenvolvimento e crescimento vegetal, e uma das melhores maneiras de relacioná-la ao desenvolvimento é por meio do uso do sistema de unidades térmicas ou graus-dia. O uso de graus-dia admite uma relação linear entre o acréscimo de temperatura do ar e a taxa de desenvolvimento a qual, apesar das restrições, permite determinar a temperatura-base ou mesmo a duração das fases fenológicas das culturas (CAMARGO, 1987).

Diferentes autores têm utilizado o índice heliotérmico de Geslin (MотA, 1969; CunHA et al., 1997 e CunHa et al., 1999) para estimar a duração das fases fenológicas das culturas para os cereais de inverno, principalmente os cultivados no Sul do País.

Em relação ao triticale, poucos dados sobre sua bioclimatologia são encontrados na literatura. WENDT e BAIER (1982), em caracterização bioclimática realizada em Passo Fundo (RS), relatam que os triticales apresentam o subperíodo emergênciaespigamento curto, sendo classificados como precoces e superprecoces. RoYo e BlANCO (1999), comparando genótipos de triticale na Espanha, mostraram que os tipos de inverno necessitam 109 graus-dia a mais que os de verão para atingir a taxa máxima de acúmulo de massa seca. Portanto, o objetivo deste trabalho foi determinar a temperatura-base e as necessidades térmicas de diferentes cultivares de triticale e a relação entre a duração do período emergência-maturação (colheita) e a temperatura do ar.

\section{MATERIAL E MÉTODOS}

Os dados fenológicos do triticale utilizados no estudo foram obtidos em experimentos desenvolvidos em área experimental do Pólo de Desenvolvimento Regional do Sudoeste Paulista, localizado no município de Capão Bonito, São Paulo (latitude: $24^{\circ}$ $00^{\prime}$ ' ; longitude: $48^{\circ} 22^{\prime} \mathrm{W}$; altitude: $702 \mathrm{~m}$ ).

Os genótipos de triticale avaliados foram CEP 15 (Panda) de ciclo superprecoce (120 dias); IAC 3 (Banteng) de ciclo precoce (121 a 130 dias) e IAC 2 (Tarasca 87) de ciclo médio (131 a 140 dias). As observações das datas de emergência e colheita (maturação) foram feitas, com quatro repetições, para diferentes épocas de semeaduras consecutivas e espaçadas de 20 a 30 dias, iniciando durante a primeira quinzena de abril, durante os anos agrícolas de 1992 a 1995.

Os dados de temperatura máxima e mínima do ar foram obtidos no posto meteorológico localizado a aproximadamente $500 \mathrm{~m}$ da área experimental.

O cálculo da soma térmica, em graus-dia, foi feito com base em:

$$
G D=\sum_{i=1}^{n}\left(\frac{T \max +T \min }{2}-T b\right)
$$


em que: GD, é o total de graus-dia acumulado; Tmax é a temperatura do ar máxima diária $\left({ }^{\circ} \mathrm{C}\right) ;$ Tmin é a temperatura do ar mínima diária (C); Tb é a temperatura-base $\left({ }^{\circ} \mathrm{C}\right)$; n é o número de dias do período de semeadura-colheita.

A determinação da temperatura-base para as variedades de triticale foi feita utilizando-se os métodos da menor variabilidade, proposto por ARNOLD (1959) e pela combinação dos métodos do desenvolvimento relativo e de regressão linear, como proposto por BRUNINI et al.(1976) e GBUR et al. (1979).

De acordo com ARnold (1959), para determinação da temperatura-base, são escolhidas a priori, valores de temperaturas que vão ser utilizadas para cálculo das somas térmicas. Posteriormente, determina-se o desvio-padrão (Sdd), em graus-dia, sendo a temperatura-base da cultivar aquela que corresponder ao menor valor de desvio-padrão (Sd), em dias, calculado como segue:

$$
\mathrm{Sd}=\mathrm{Sdd} /(\mathrm{Xt}-\mathrm{Tb})
$$

em que:, Xt é a temperatura média para toda a série de plantios analisados.

As temperaturas utilizadas para determinação da temperatura-base foram: $0,2,4,6,8,10,12,14,16$, 18 e $20^{\circ} \mathrm{C}$.

O método do desenvolvimento relativo (DR), segundo BRUNINI et al. (1976) e GBUR et al., (1979), para a estimativa da temperatura-base é feito da seguinte maneira:

$$
\mathrm{DR}=\mathrm{a}+\mathrm{b} . \text { Tmed }
$$

em que: Tmed é a temperatura média $\left({ }^{\circ} \mathrm{C}\right)$ e DR é calculado por:

$$
D R=\frac{100}{n}
$$

quando DR for igual a zero, Tmed será igual a $\mathrm{Tb}$; sendo obtida por:

$$
T b=\frac{-a}{b}
$$

a e b são os coeficientes linear e angular da regressão linear simples.

\section{RESULTADOS E DISCUSSÃO}

Os resultados obtidos de temperatura-base, soma térmica e duração do ciclo para as diferentes cultivares de triticale, cultivadas em Capão Bonito, são apresentados nas Figuras 1 a 3 e Tabelas 1 e 2.

\section{Temperatura-base}

Na figura 1 é apresentada a determinação da temperatura-base pelo método da menor variabilidade.

Os valores obtidos de temperatura-base foram: 8,8 e $10^{\circ} \mathrm{C}$, respectivamente para as cultivares, CEP15, IAC 3 e IAC 2. Verificou-se que a diferença nos valores do desvio-padrão, em dias, variou bem pouco em torno da temperatura-base em determinação, principalmente para as cultivares CEP 15 e IAC 3, podendo dificultar sua melhor caracterização.

A determinação da temperatura-base para as cultivares de triticale- CEP 15, IAC 3 e IAC 2 - foi feita, também, pelo método do desenvolvimento relativo. $\mathrm{Na}$ figura 2, são apresentados os valores de Tb obtidos, que foram: $8,5^{\circ} \mathrm{C}$ para CEP $15 ; 8,3{ }^{\circ} \mathrm{C}$ para o IAC $3 \mathrm{e}$ $10,5^{\circ} \mathrm{C}$ para IAC 2 .

Os valores determinados de temperatura-base pelos dois métodos foram muito próximos, mostrando a viabilidade de sua utilização em cálculos de somas térmicas para as cultivares de triticale.

Observou-se que para as cultivares de triticale de ciclo superprecoce e precoce (CEP 15 e IAC 3) os valores obtidos de temperatura-base foram próximos de $8{ }^{\circ} \mathrm{C}$, enquanto para a cultivar de ciclo médio (IAC 2) a temperatura-base foi superior $\left(10^{\circ} \mathrm{C}\right)$.

Os valores de temperatura-base encontrados são superiores ao valor de $4,4{ }^{\circ} \mathrm{C}$ utilizado por Nuttonson (1955) e Peterson (1965) para o trigo. Segundo o último autor, essa temperatura-base fornece resultados mais consistentes de soma térmica e tem sido utilizada normalmente nos países norteamericanos. Além disso, SHAYKewich (1995) afirmou que após o trabalho original de Nuttonson, diferentes valores de temperatura-base têm sido usados para o trigo e cevada, variando entre 0 e $4{ }^{\circ} \mathrm{C}$.

Verificou-se que as valores de temperaturabase obtidos para os cultivares de triticale $\left(8\right.$ e $\left.10^{\circ} \mathrm{C}\right)$ foram próximos aos citados por Abreu e CAMPBell (1997), ou seja $12,1{ }^{\circ} \mathrm{C}$, utilizado na previsão de época de maturação do trigo em Portugal.

\section{Necessidades térmicas}

As necessidades térmicas para diferentes cultivares de triticale estão apresentadas na Tabela 1. Para 'CEP 15' e 'IAC 3' utilizou-se a temperaturabase de $8{ }^{\circ} \mathrm{C}$, sendo obtido um acúmulo de graus-dia para o período emergência-maturação, de 1.172 e 1.263 graus-dia, respectivamente. Para IAC 2, em temperatura-base de $10^{\circ} \mathrm{C}$, o total de graus-dia médio obtido foi de 1.048 . 

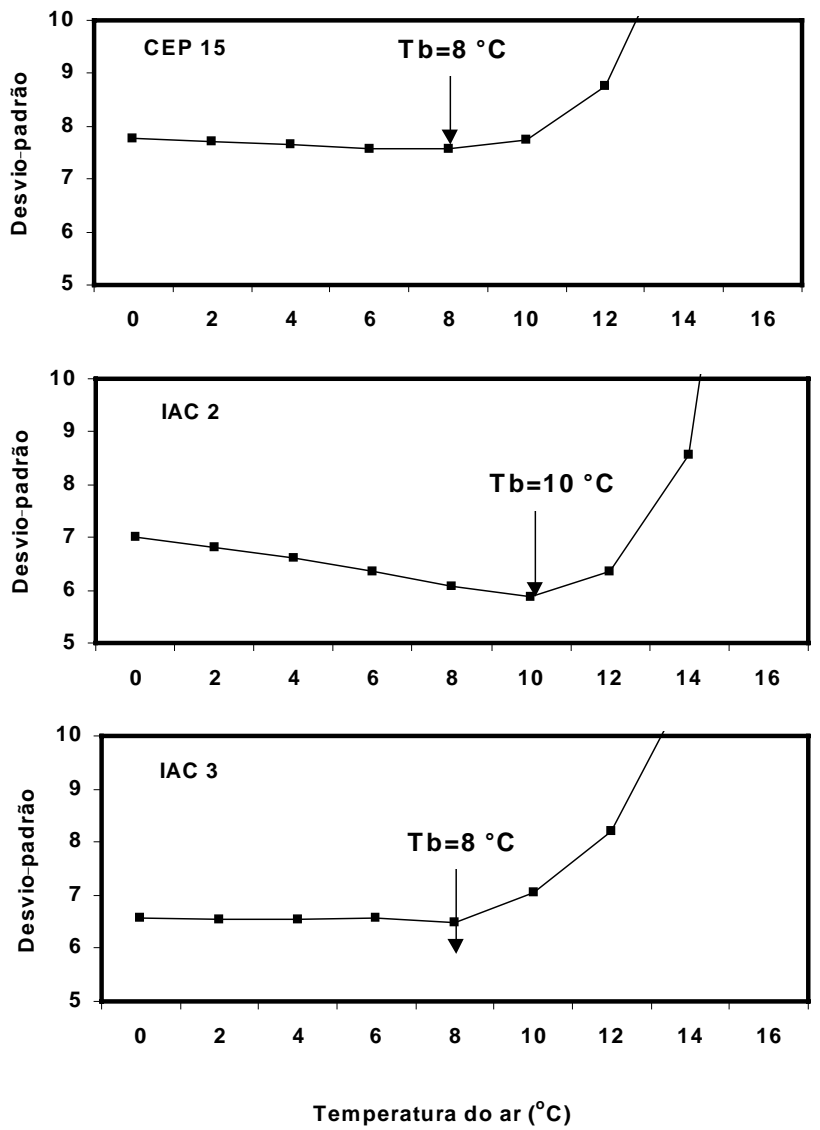

Figura 1. Determinação da temperatura-base para o período emergência-maturação, pelo método da menor variabilidade, para cultivares de triticale em Capão Bonito (SP).
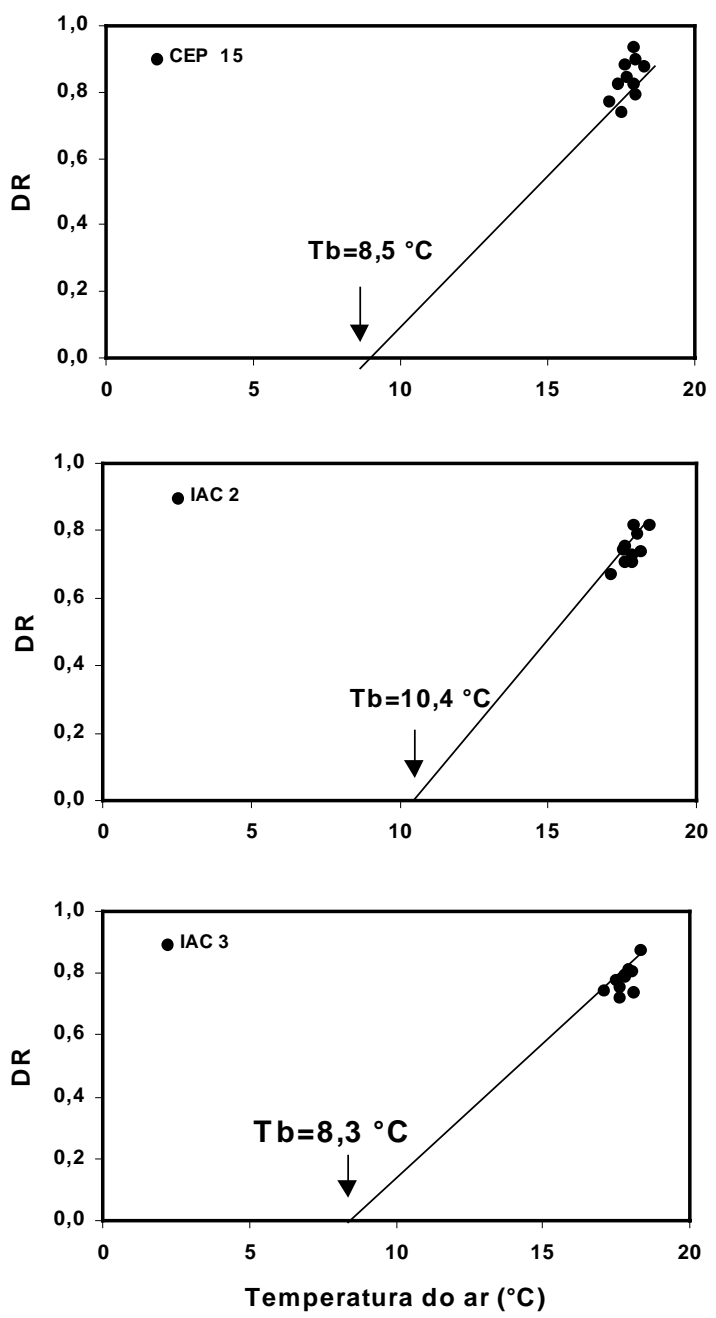

Figura 2. Determinação da temperatura-base do período emergência-maturação, pelo método da regressão linear, para cultivares de triticale, em Capão Bonito (SP).

Tabela 1. Somas térmicas, em graus-dia, no período emergência-maturação para cultivares de triticale, em diferentes épocas de semeadura, em Capão Bonito (SP)

\begin{tabular}{lcccr}
\hline Ano & $\begin{array}{c}\text { Data de } \\
\text { semeadura }\end{array}$ & $\begin{array}{c}\text { CEP 15 } \\
\left(\mathrm{Tb}=8^{\circ} \mathrm{C}\right)\end{array}$ & $\begin{array}{c}\text { IAC 3 } \\
\left(\mathrm{Tb}=8^{\circ} \mathrm{C}\right)\end{array}$ & $\begin{array}{c}\text { IAC 2 } \\
\left.\mathrm{Tb}=10^{\circ} \mathrm{C}\right)\end{array}$ \\
\hline 1992 & $23 / 4$ & 1.149 & 1.276 & 1.010 \\
& $18 / 5$ & 1.146 & 1.222 & 1.018 \\
1993 & $9 / 6$ & 1.090 & 1.212 & 966 \\
& $7 / 4$ & 1.115 & 1.260 & 1.110 \\
1994 & $24 / 4$ & 1.183 & 1.183 & 1.071 \\
& $4 / 4$ & 1.206 & 1.261 & 1.072 \\
1995 & $26 / 4$ & 1.297 & 1.363 & 1.09 \\
& $4 / 4$ & 1.073 & 1.370 & 1.020 \\
& $26 / 4$ & 1.274 & 1.274 & 1.036 \\
\hline Média & $2 / 6$ & 1.189 & 1.189 & 1.048 \\
Desvio-padrão & & 1.172 & 1.263 & 45 \\
\hline
\end{tabular}


Nenhum trabalho foi encontrado que mostrasse a soma térmica para o triticale, porém, NutTonson (1955) afirma que o trigo de primavera necessita para completar o ciclo cerca de 1.130 grausdia nos Estados Unidos e na Rússia e cerca de 980 graus-dia no Canadá.

\section{Duração do ciclo}

A duração do período emergência-maturação (colheita) é apresentada na tabela 2 e sua relação com a temperatura média do ar, na figura 3. A duração do período emergência-maturação obtida foi, em média, 120, 128 e 134 dias, respectivamente, para CEP 15, IAC 3 e IAC 2, podendo ser afetada pela ocorrência de secas, encurtando, muitas vezes, o ciclo da cultura, com quebra na produtividade. A temperatura do ar tem influência na duração do ciclo por torná-lo mais longo em plantios efetuados em épocas mais frias. Pode ser verificado pela figura 2 que a cultivar CEP 15 obteve duração de ciclo variando de 107 a 135 dias para temperaturas médias no ciclo de 17,1 a $18,3{ }^{\circ} \mathrm{C}$, enquanto para IAC 3 a variação no ciclo foi de 114 a 139 dias, e para IAC 2, de 122 a 149 dias.

Tabela 2. Duração (dias) período emergência-maturação para diferentes anos agrícolas, épocas de semeadura e cultivares de triticale em Capão Bonito (SP)

\begin{tabular}{|c|c|c|c|c|c|c|c|c|c|c|}
\hline \multirow{2}{*}{ Ano } & \multirow{2}{*}{$\begin{array}{c}\text { Data de } \\
\text { semeadura }\end{array}$} & \multicolumn{3}{|c|}{ CEP 15} & \multicolumn{3}{|c|}{ IAC 3} & \multicolumn{3}{|c|}{ IAC 2} \\
\hline & & E-F & F-M & E-M & E-F & F-M & E-M & E-F & F-M & E-M \\
\hline \multirow[t]{3}{*}{1992} & $23 / 4$ & 47 & 71 & 118 & 56 & 76 & 132 & 57 & 75 & 132 \\
\hline & $18 / 5$ & 51 & 70 & 121 & 58 & 70 & 128 & 63 & 71 & 134 \\
\hline & $9 / 6$ & 55 & 58 & 113 & 55 & 68 & 123 & 61 & 61 & 122 \\
\hline \multirow[t]{2}{*}{1993} & $7 / 4$ & 41 & 70 & 111 & 41 & 86 & 127 & 58 & 83 & 141 \\
\hline & $24 / 4$ & 49 & 80 & 129 & 63 & 71 & 134 & 64 & 85 & 149 \\
\hline \multirow[t]{2}{*}{1994} & $4 / 4$ & 42 & 79 & 121 & 48 & 78 & 126 & 56 & 81 & 137 \\
\hline & $26 / 4$ & 49 & 86 & 135 & 62 & 77 & 139 & 73 & 68 & 141 \\
\hline \multirow[t]{3}{*}{1995} & $4 / 4$ & 46 & 61 & 107 & 56 & 79 & 135 & 63 & 72 & 135 \\
\hline & $26 / 4$ & 56 & 70 & 126 & 61 & 63 & 124 & 67 & 59 & 126 \\
\hline & $2 / 6$ & 63 & 51 & 114 & 62 & 52 & 114 & 63 & 59 & 122 \\
\hline \multicolumn{2}{|c|}{ Média } & 49,9 & 69,6 & 119,5 & 56,2 & 72,0 & 128,2 & 62,5 & 71,4 & 133,9 \\
\hline \multicolumn{2}{|c|}{ Desvio-padrão } & 6,7 & 10,7 & 8,7 & 7,0 & 9,6 & 7,2 & 5,0 & 9,7 & 8,8 \\
\hline
\end{tabular}

E-F: emergência-florescimento; F-M: florescimento-maturação; E-M: emergência-maturação (colheita).
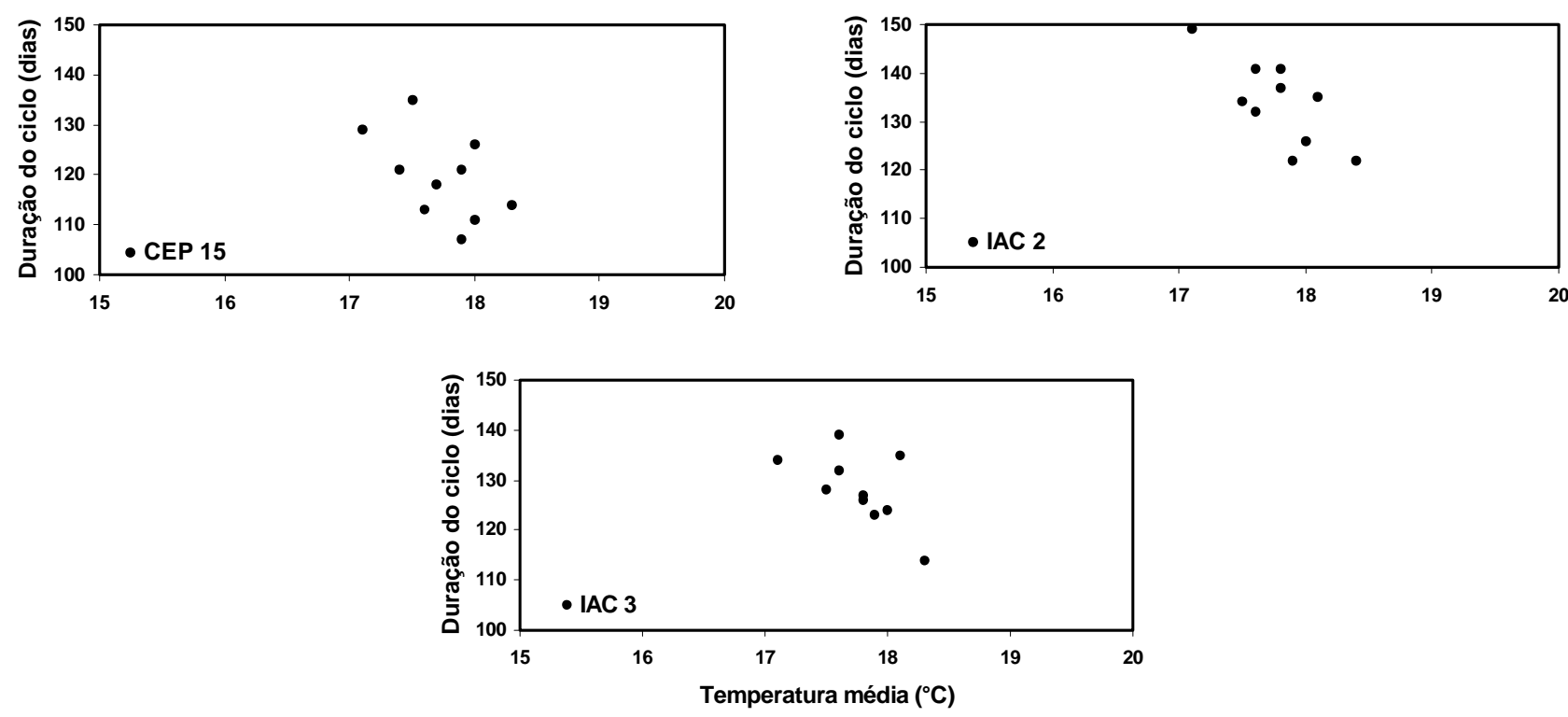

Figura 3. Relação entre a duração do ciclo de diferentes cultivares de triticale e a temperatura em Capão Bonito (SP). 
As durações médias do subperíodo emergência-florescimento (Tabela 2) observadas foram de 50, 56 e 63 dias respectivamente para as cultivares CEP 15, IAC 3 e IAC 2, enquanto a duração do subperíodo florescimento-maturação foi, em média de 70 dias para as três cultivares de triticale. Esse fato também foi observado por Mundstock (1983) que ressalta ser a ação da temperatura nos cereais de inverno mais efetiva, principalmente na duração do período compreendido entre a emergência e o florescimento. Os valores obtidos são inferiores aos relatados por GUEDES-PINTO et al., (1986), que obtiveram em Portugal, para variedades provenientes do CIMMYT, 150 dias até a floração e 250 dias até a maturação.

\section{CONCLUSÕES}

1. Os valores de temperatura-base determinados para as diferentes cultivares de triticale foram: $10^{\circ} \mathrm{C}$ para IAC 2 e $8{ }^{\circ} \mathrm{C}$ para CEP 15 e IAC 3 . Não se notou diferença nos valores de temperaturabase obtidos pelos diferentes métodos utilizados, ou seja, o da menor variabilidade e o do desenvolvimento relativo.

2. As somas térmicas, expressas em graus-dia, obtidas, como média do período analisado, foram: $1.048,1.172$ e 1.263, respectivamente, para IAC 2, CEP 15 e IAC 3.

3. A duração do subperíodo emergênciaflorescimento variou entre 50 e 63 dias, em função das cultivares, enquanto a do subperíodo florescimentomaturação foi, em média, de 70 dias para as três cultivares analisadas. A duração total do ciclo do triticale nos plantios efetuados em épocas mais frias aumentou em virtude da diminuição da temperatura do ar.

\section{REFERÊNCIAS}

ABREU, J.P.M.; CAMPBELL, G.S. Spring wheat development under mediterranean conditions: 1 . Prediction of anthesis and maturiy. Revista de Ciências Agrárias, Lisboa, v.20,n.2, p.17-32, 1997.

ARNOLD, C.Y. The determination and significance of the basetemperature in a linear heat unit system. Proceedings of the American Society for Horticultural Science, Alexandria, v.74, p.430-445, 1959.

BAIER, A.C. Potencial do triticale no Brasil. In: REUNIÃO BRASILEIRA DE TRITICALE, 4., 1992, Chapecó. Anais... Chapecó: Epagri, 1995. p.8-23.
BRUNINI, O.; LISBÃO, R.S.; BERNARDI, J.B.; FORNASIER, J.B.; PEDRO JÚNIOR, M.J. Temperatura-base para alface cultivar "White Boston", em um sistema de unidades térmicas. Bragantia, Campinas, v.35, n.19, p. 213-219, 1976.

CAMARGO, M.B.P.; BRUNINI, O.; MIRANDA, M.A.C. Temperatura-base para cálculo dos graus-dia para cultivares de soja em São Paulo. Pesquisa Agropecuária Brasileira, Brasília, v.22, n.2, p.115-121, 1987.

CUNHA, G.R.; SCHEEREN, P.L.; RODRIGUES, O.; LUCA, L.J.A.; FIORINI, M.C.; SILVA, C.F.L. Bioclimatologia de trigos sul-brasileiros. Revista Brasileira de Agrometeorologia, Santa Maria, v.5, n.2, p.195-198, 1997.

CUNHA, G.R.; SILVA, M.S.; ARIAS, G. DALMAGO, G.A. Caracterização bioclimática de cevadas brasileiras. Revista Brasileira de Agrometeologia, Santa Maria, v.7, n.2, p.201206, 1999.

FELICIO, J.C.; CAMARGO, C.E.O.; CASTRO, J.L.; CAMARGO, M.B.P. Épocas de semeadura de triticale em Capão Bonito, SP. Pesquisa Agropecuária Brasileira, Brasília, v.34, n.12, p.21932202, 1999.

FELICIO, J.C.; CAMARGO, C.E.O.; FERREIRA FILHO; A.W.P.; CASTRO, J.L. Comportamento de cultivares de triticale no Estado de São Paulo no período de 1985 a 1987. Pesquisa Agropecuária Brasileira, Brasília, v. 25, n.8, p.1143-1149, 1990.

FELICIO, J.C.; CAMARGO, C.E.O.; PEZZINATO, A.; CASTRO, J.C.; GALLO, P.B. Comportamento agronômico e avaliação tecnológica de genótipos de triticale no Estado de São Paulo de 1988 a 1989. Pesquisa Agropecuária Brasileira, Brasília, v. 28, n.3, p.281-294, 1993.

GBUR, E.E.; THOMAS, G.L.; MILLER, F.R. Use of segmented regression in determination of the base-temperature in heat accumulation models. Agronomy Journal, Madison, v.71, p.949-953, 1979.

GUEDES-PINTO, H.; CARNIDE, O.; CARNIDE, V. Dois anos de ensaios de adaptação com triticales em Trás-os-Montes. Melhoramento, Elvas, v.27, p.49-89, 1986.

MOTA, F.S.; GOEDEST, C.D. Características bioclimáticas dos trigos sul-brasileiros. Pesquisa Agropecuária Brasileira, Brasília, v.4, p.79-87, 1969.

MUNDSTOCK, C.M. Cultivo dos cereais de estação fria. Editora NBS: Porto Alegre (RS), 1983. 265p.

NUTTONSON, M.Y. Wheat-climate relationships and use of phenology in ascertaining the thermal and photo-thermal requirements of wheat. Washington: American Institute of Crop Ecology, 1955. 388p.

PETERSON, R.F. Wheat: botany, cultivation and utilization. World Crops Books. London: Leonard Hill Books, 1965. 421p.

RODRIGUES, O. Manejo de trigo: bases ecofisiológicas. In: CUNHA, G.R.; BACALTCHUK,B.(Orgs.). Tecnologia para produzir trigo no Rio Grande do Sul. Porto Alegre: EMBRAPA, 2000. p.120-155. (Série Culturas n 2) 
ROYO, C.; BLANCO, R. Growth analysis of five spring and five winter triticale genotypes. Agronomy Journal, Madison, v.91, p.305-311, 1999.

SHAYKEWICH, C.F. An appraisal of cereal crop phenology modelling. Canadian Journal of Plant Science, Ottawa, v.75, n.2, p.329-341, 1995.

TAGLIARI, P.S. Triticale uma nova (e boa) alternativa de inverno. Agropecuária Catarinense, Florianópolis, v.9, n.1, p.21-23, 1996
WENDT, W.; BAIER, A.C. Caracterização bioclimática de alguns genótipos de triticale. In: REUNIÃO NACIONAL DE TRIGO, 12, 1982, Cascavel. PR. Resumos e Comunicados Técnicos... Cascavel: OCEPAR, 1982. p.21. 